\title{
Cytoarchitecure and Connectivity of the Superior Colliculus in Mouse Brain
}

\author{
Youshan Zhang \\ Department of Computer Science and Engineering, Lehigh University, Bethlehem, USA \\ Email address: \\ yoz217@lehigh.edu \\ To cite this article: \\ Youshan Zhang. Cytoarchitecure and Connectivity of the Superior Colliculus in Mouse Brain. International Journal of Biochemistry, \\ Biophysics \& Molecular Biology. Vol. 3, No. 2, 2018, pp. 30-37. doi: 10.11648/j.ijbbmb.20180302.12
}

Received: June 7, 2018; Accepted: June 26, 2018; Published: July 18, 2018

\begin{abstract}
Superior Colliculus (SC) plays a vital role in visual target selection and attention shifting; it also an important structure in studying the central nervous system. However, the cytoarchitecture and connectivity of the SC in mouse brain have not been explicitly explored. In this paper, to investigate the structural delineations and of connectivity SC, we first delineated the morphology of the SC by Nissl stain, and further explored different genes expressed in the SC. It demonstrates that gene Tpd5211 is densely expressed in the SC, which helps describe the borders of the SC. In addition, we explore the connectivity of Superior Colliculus (validating the projections from other structures to SC and examining the projections from SC to other structure). The anterograde and retrograde projection circuitries between SC and IC are particularly addressed, which indicates that the SC is involved in the visual, auditory and other somatosensory physiological activities.
\end{abstract}

Keywords: Superior Colliculus, Cytoarchitecure, Connectivity, Gene Expression, Mouse Brain

\section{Introduction}

The SC (Superior Colliculus) is a superstructure of the mammalian midbrain quadrigeminal body [1-2]. It is also known as the optic tectum, or simply "tectum" in vertebrates. The SC is a relay nucleus in the mammalian visual pathway and the primary target of the primary visual projection. It is not only an important reflex center of the visual pathway, but also involved in many physiological activities, such as auditory, somatosensory and other activities [3-5]. Therefore, the $\mathrm{SC}$ is considered to be an essential structure in the study of the central nervous system.

The SC is structurally divided into seven layers in mammals: the zonal layer (ZO), superficial gray layer (SGR), optic layer (OP), intermediate gray layer (IGR), intermediate white layer (IW), deep gray layer (DGR), and deep white layer (DW) (figure 1). The ZO, SGR and OP are three superficial layers (SL) which receive most of the afferent fibers from the retina and the visual cortex and are mainly involved in visual information formation. While IGR, IW, DGR, and DW are four deep layers (DL), which receive visual projections from cortical and subcortical regions, they also receive auditory and somatosensory projections. At the same time, it also issues motion instructions and controls the eyes and ears and head toward the significant stimuli in animals [6-9]. Therefore, the SC is considered recognized as a visual motor integration center of the central nervous system. The superficial layers receive direct visual inputs, but the deep layers receive indirect visual inputs [10-11]. The superficial layer and deep layer cells in morphology differ with respect to two main points: the superficial layer cells have short and dense but not overlapping dendrites, similar to the structure found in the perception of the neurons, with significant differences compared to the relatively long and overlapping neuronal dendrites in the deep layer. The second is that the difference of superficial layer neurons in cell size is not as significant as deep layer neurons [12].

The origin of the superior colliculus fibers is complex, there are many studies about this, and the afferent fiber mainly of SC can be classified into two categories. One is related to the visual function of the nucleus, called visual structure, and the other is independent of the visual function of nuclei, called non-visual structure. There are several different types of projections in visual and non-visual structures. The projections of visual structure include the projection from the retina to SC (retinal 
ganglion cells projecting to the superior colliculus are one of the largest sources of afferent fibers in the superior colliculus [13-15], the projection from the visual cortex to SC (in mammals, the visual cortex is generally divided into the primary visual area (area 17), the secondary visual area (area 18) and the third area (area 19). The primary visual area is mainly reacted to the contrast of retina graphics and movements, secondary visual area and the third area is processed, analyzed and integrated the visual information [16-17]. The lateral geniculate nucleus is a relay station of the visual pathway in the thalamus, the projection from lateral geniculate nucleus to SC is composed of several layers of cells and fibers. The projections of the non-visual structure include the projection from parabigeminal nucleus $(\mathrm{Pbg})$ to $\mathrm{SC}$, the projection from Substantia nigra (Sn) to SC, the projection from Inferior colliculus (IC) to $\mathrm{SC}$ and the projection from the dorsal nucleus of the lateral lemniscus (Dll) to SC [18-19]. The projections from the efferent fibers include the projection from the SC to the lateral geniculate nucleus ( $\mathrm{LGN}$ ), the projection from the $\mathrm{SC}$ to $\mathrm{Pbg}$, and the projection from the SC to the auditory cortex [20-22]. Additionally, the superior colliculus also sends fibers to the nucleus of the $\mathrm{Sn}$, the anterior tectum, the superior nucleus of the thalamus, the nucleus of the thalamus, and other structures. Moreover, there is a complex fiber connection between both sides of the colliculus.

The purpose of this study is two-fold. First, the study aims to investigate the structural delineations of SC, especially the sub-regions which can reflect the functions. Secondly, this paper validates the projections from other structures to SC and examines the projections from SC to other structures. The hypothesis is that gene expression in the SC can also be found in other structures to illustrate the role the current gene. In addition, the other hypothesis is the projections of $\mathrm{SC}$ can demonstrate the connectivity between SC and different structures.

\section{Method}

To delineate the cytoarchitecture of SC, two public online sources are used: the Allen Brain Atlas (ABA) and the Mouse Connectome Project [23]. The brainmaps.org website is more used to explore sub-regions and the gene expression in the SC, and mouseconnectome.org website is used to find connectivity information about SC.

\subsection{Nissl Staining}

Based on the Allen Brain Atlas, six coronal slices (83, 86, 88, 91, 94 and 103) and one sagittal slice representative images have been selected to illustrate the different regions in the SC.

\subsection{Gene Expression}

Because not all the listed gene in the ABA can be clearly observed in the SC, eight representative genes (Gabrr1,
Tpd5211, LOC433228, Mgll, Lats2, Dgkh, Cpne5 and LOC433258) are selected.

\subsection{Connectivity}

Both the ABA and the mouseconnectome website are used to explore the connectivity, and several tracers are used including AAV, Ctb555, PHAL and fg tracer.

\section{Results and Discussion}

\subsection{Cytoarchitecture}

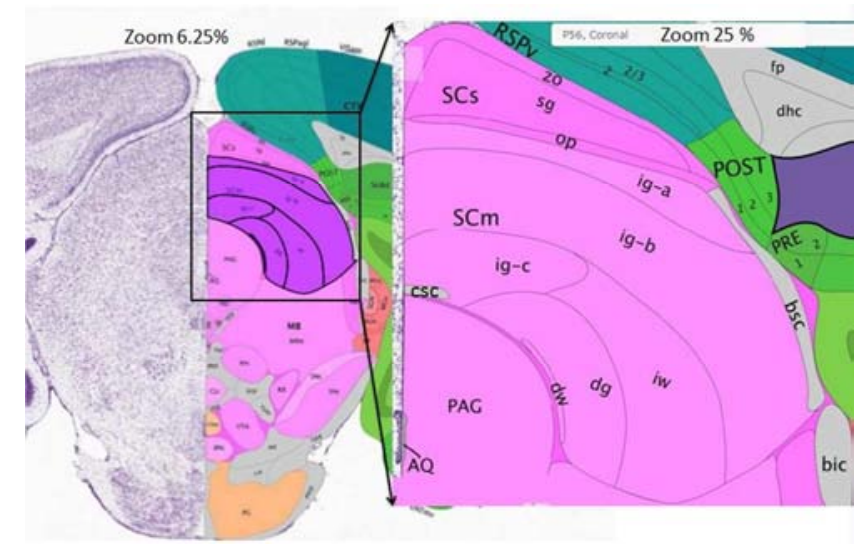

Figure 1. The location and sub-regions of the SC (Coronal view, atlas image 90). SC has different sub-regions (include the seven layers: ZO layer, SGR layer, OP layer, IGR layer, IW layer, DGR layer, and DW layer).

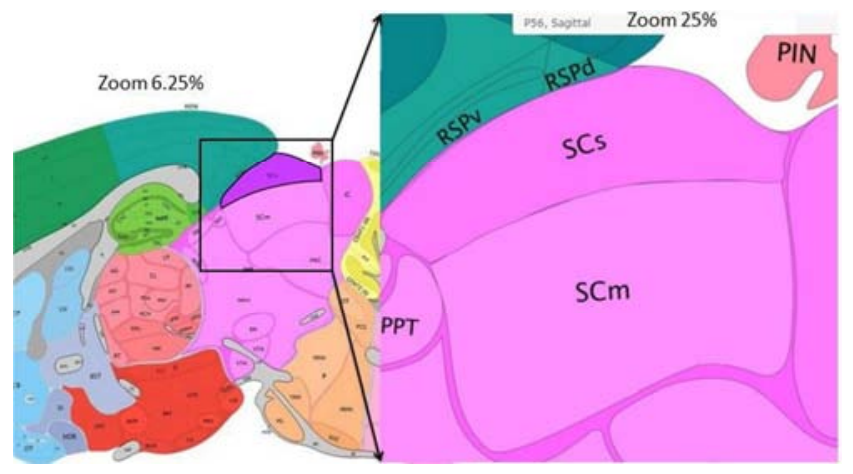

Figure 2. The location and sub-regions of the SC (Sagittal view, atlas image 15).

As noted from figure 1 and figure 2, the different regions of the SC in the coronal view can be clearly distinguished. In addition to the seven layers in the SC, it shows that the SC can be divided into sensory related (SCs), motor related (SCm) and two fiber tracts (brachium of the superior colliculus (bsc) and superior colliculus commissure (csc)). The sensory related structures in SC can be subdivided into ZO layer, SGR layer and OP layer, which are also called superficial layers. The motor related structures in SC can also be classified in to IGR layer, IW layer, DGR layer, and DW layer, which are also called deep layers. 


\subsubsection{Nissl Staining}

Table 1. Cytoarchitecture of the Superior Colliculus from Nissl stain (Macaca mulatta) overlay on the ABA.

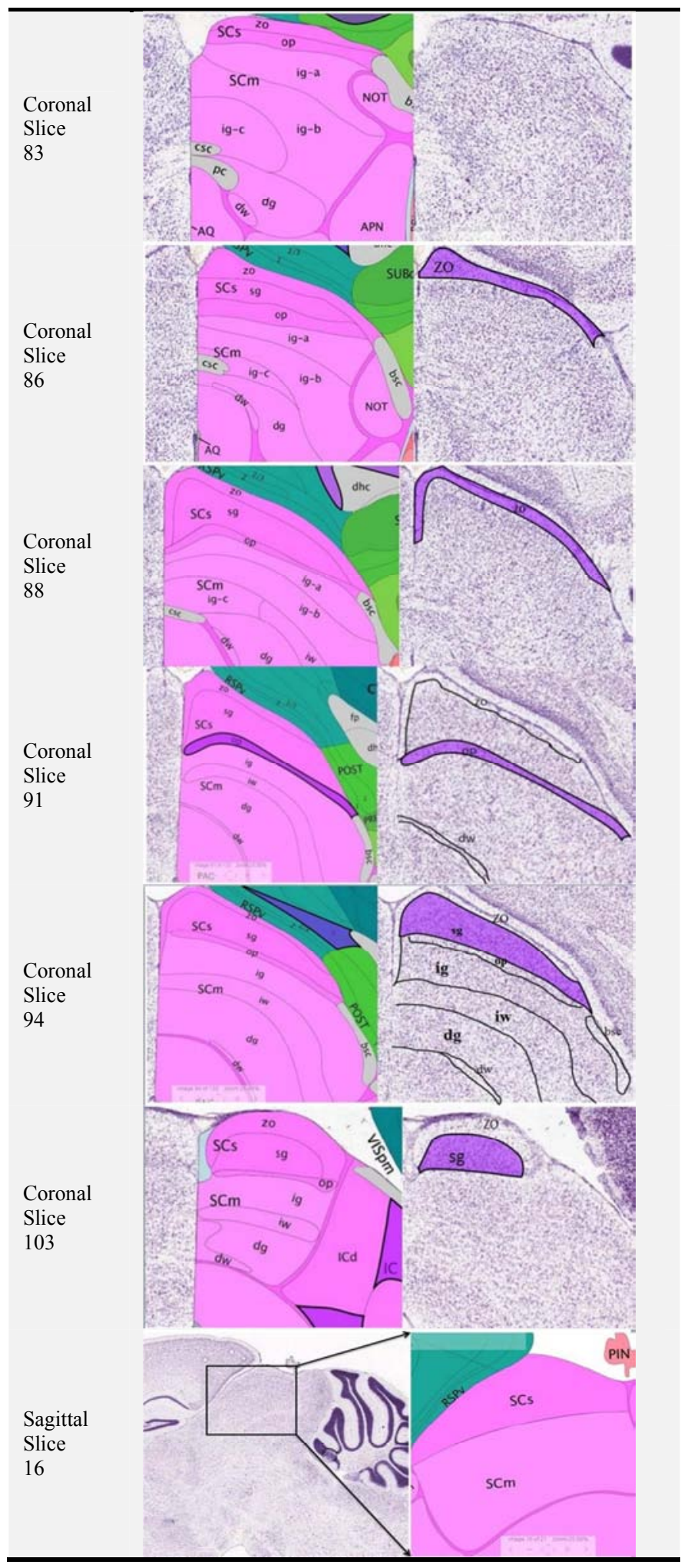

Table 1 shows the location and cytoarchitecture of SC. Six Coronal slices $(83,86,88,91,94$ and 103) and one sagittal slice representative images have been selected to illustrate the different regions in the SC. Each slice contains two images; the left is named SC sub-regions and the right is the corresponding Nissl stain images. The purple highlight on the right Nissl stain images is the region which can be identified easily. In addition, the manual delineated lines are the boundaries between different sub-regions. The Nissl stain in slice 83 can hardly distinguish the different sub-regions in the SC; the slice 86 and 88 can easily find the ZO layer in the superficial layers; the slice 91,94 and 103 can identify more regions, especially slice 94 , which can substantially identify all the sub-regions in the SC. It is impossible to identify the seven sub-regions in the sagittal slice 16, since these sub-regions in $\mathrm{SCs}$ and $\mathrm{SCm}$ are completely compacted with each other.

The superior colliculus is not a homogenous structure. The superficial layers (ZO, SGR and OP) receive information from visual systems, while the deep layers (IGR, IW, DGR, and DW layer) receive visual, auditory, somatosensory signal from cortex, and inputs from the frontal eyes fields. Therefore, the SC is almost multimodal and associational [10-24]. As shown in the six slices, the superficial layers are significantly different from the deep layers. The area of deep layers is obviously larger than the superficial layers, in that they are more pronounced with a higher cell density in the deep layer compared to the superficial layers. These two layers can be divided by the OP layer which is a slender region. The deep layers are compacted below the OP layer. The sub-regions in SC are actually difficult to distinguish either the superficial layers or the deep layers; only small number of Nissl stain image can be identified, for example, slice 83 is difficult to identify the seven layers in the SC. Besides, two sub-regions (bsc and csc) are not further explored, since these two fibers tracts are not the region of interests (ROIs). In the slice 86 and 88 , the first layer $\mathrm{ZO}$ can be facile identified, since this layer is just below the Retrosplenial area (RSP). The OP and DW layers have relatively low cell density than the other 5 layers. In addition, SGR has the highest number of the cell density among these regions.

\subsubsection{Gene Expression}

Table 2 illustrates the distinctively expressed gene in the SC. Eight genes have been selected to show the differentiation in the SC. There are 21 genes in the mouse.brain-map.org, but some of the gene expression is not visible in the images, so eight representative genes (Gabrr1, Tpd5211, LOC433228, Mgll, Lats2, Dgkh, Cpne5 and LOC433258) are selected. Four genes (Tpd5211, Dgkh, Cpne5 and LOC433258) are densely expressed within the $\mathrm{SC}$, and the other four genes are sparsely expressed in the SC. 
Table 2. Gene expression of the SC (Macaca mulatta), the left column is the expressed gene and three different slices about that gene in the SC region.

Gabrr1 Slice: 39 ,

41 and 43

Tpd5211 Slice: 71,

74 and 80

LOC433228

Slice: 70,75 and 77

Mgll Slice:36, 39

and 42

Lats2 Slice:37,39,

and 40

Dgkh Slice: 37,39

and 41

Cpne5 Slice: 54, 56 and 61

LOC433258 Slice:

34, 36 and 39
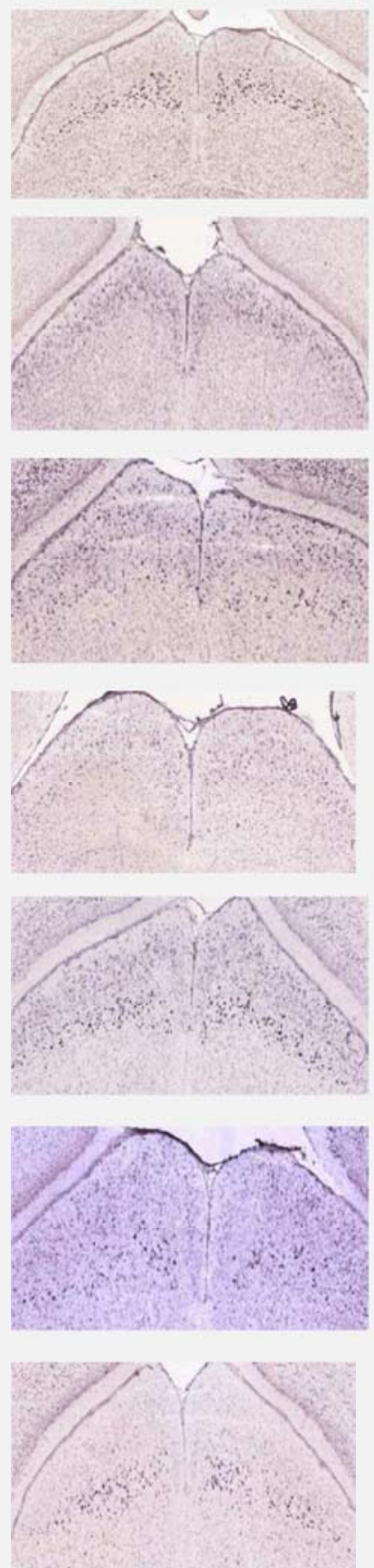
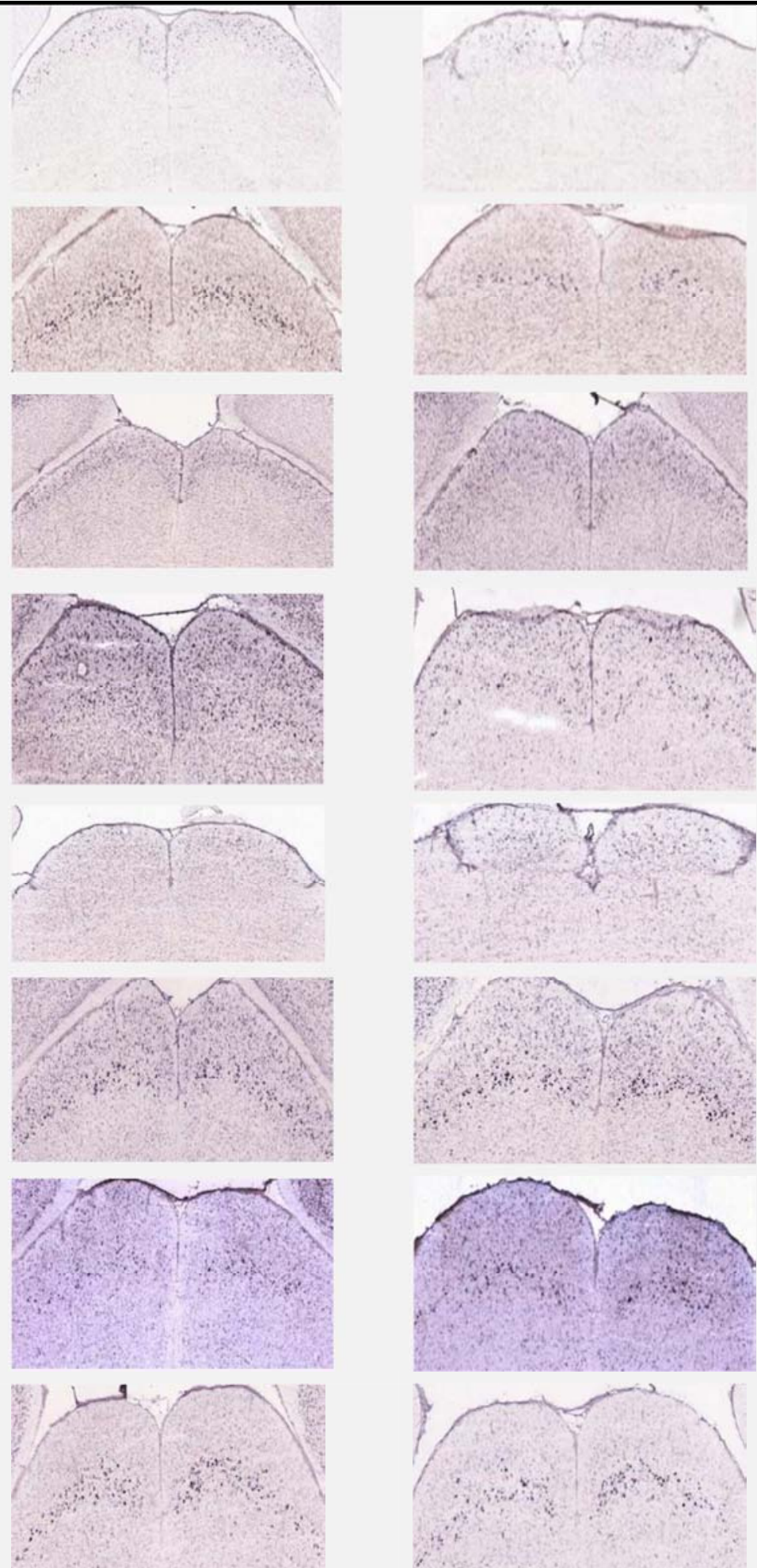
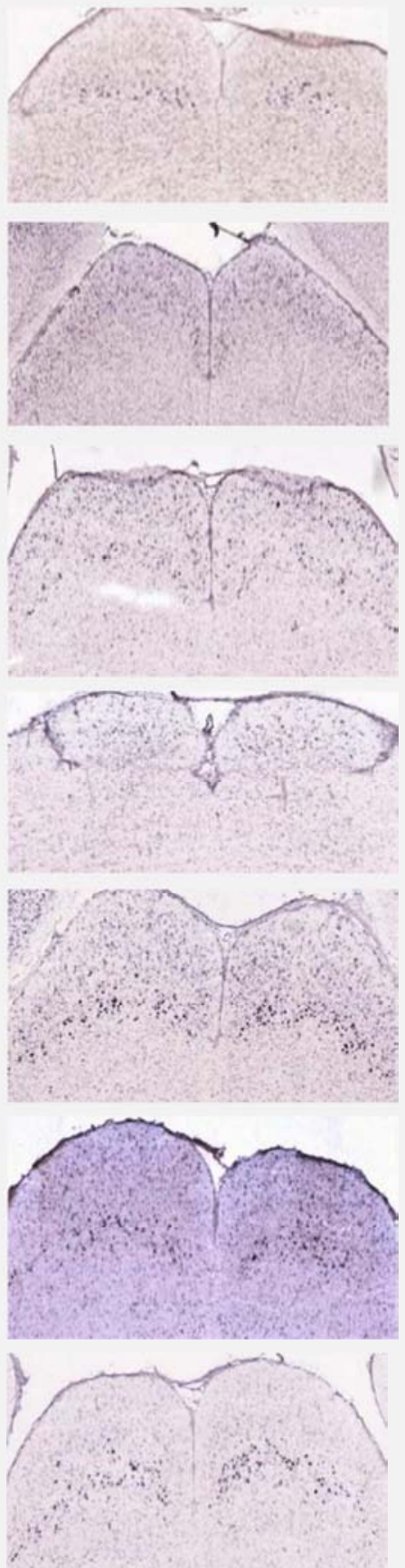

The name of Tpd5211 gene is tumor protein D52-like 1, it also can be found to be highly expressed in thalamus, and it is a protein coding gene. As shown in table 2, the Tpd5211gene has a number of dense black dots in the IGR layer, which means that there may be more cell proliferation in IGR layer [25]. The name of Dgkh gene is diacylglycerol kinase Eta. It is also a protein coding gene which encodes for the diacylglycerol kinase (DGK) enzyme and is densely expressed in the OP layer (table 2), and densely expressed in several structures (Isocortex, Olfactory areas, Hippocampal formation, Cortical subplate, Striatum, Thalamus, Midbrain and Cerebellum). It has been observed that the DGKH gene is associated with several complex diseases include Bipolar Disorder and Schizophrenia. Therefore, there are some 
changes in the structures that the DHKH expressed when the disease symptoms are manifested [26]. The name of Cpne5 gene is copine $\mathrm{V}$, it is one of the genes which encodes the calcium-dependent protein, a protein that can regulate molecular events and play role in calcium-mediated intracellular processes and the formation of the dendrites (RefSeq, Sep 2015). The gene Cpne5 is expressed in the OP layer and can also be found in other structures (Isocortex, Olfactory areas, Hippocampal formation, cortical subplate, and Pallidum) which have calcium-dependent protein. The name of LOC433258 is attractin-like 1. It is highly expressed in OP and SGR layers, and can also be found in other structures including Isocortex, Olfactory areas, Hippocampal formation, Cortical subplate, Thalamus, Midbrain. For the other four genes (Gabrr1, LOC433228, Mgll and Lats2) that are sparsely expressed in the SC also play an important role in the protein expression. For example, the Gabrr1 (gamma-aminobutyric acid (GABA) $\mathrm{C}$ receptor, subunit rho 1), a locus for GABA receptor subunit encoding, has been identified to be slightly expressed in the superficial layers. GABA is the major inhibitory neurotransmitter in the mammalian brain. On the whole, the superior colliculus has a relative higher volume of GABA, while the GABA volume in SGS layer is highest in the central nervous system. GABAc receptors are first found in the retina, but in a few of the brain regions that contain this receptor, SGS is the most strongly labeled [28].

There are still thirteen genes expressed in the SC which are not as specifically explored as the above eight genes, but these thirteen genes are also play a role in the $\mathrm{SC}$, this research does not cover these genes.

\subsection{Connectivity}

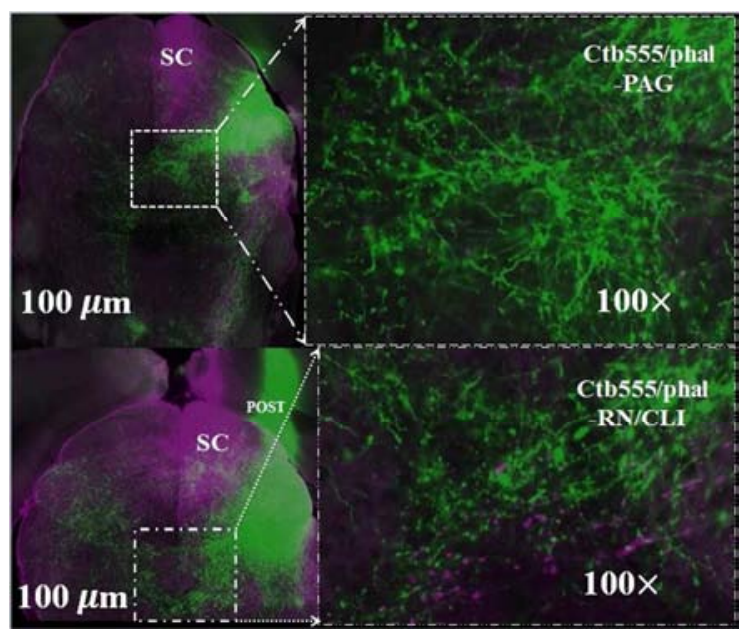

Figure 3. The anterograde projections are from the SC to different regions of the mouse brain using mouseconnectome. org. It used the Ctb555 and phal as the tracers. The left image is composed of injection sites in the SC to other structures, and the right image is the zoom in (100\% larger) projected structures (PAG: Periaqueductal Gray, RN: Red Nucleus, CLI: Central linear nucleus raphe).

Table 3 illustrates the anterograde projection from SC to other regions based on the Allen Brain Atlas. The left column displays the projection structures, and the right column shows the four different slices overlaid on the ABA. There are six representative projections are shown in the table 3 , and more projections are described on connectivity. brain-map. org. The sensory related SC (superficial layers) can project to motor related SC (deep layers), retrosplenial area (RSPv, ventral part), retrosplenial area (RSPd, dorsal part), and inferior colliculus (IC). The motor related SC (deep layers) can project to SCs, IC, RSP, Midbrain reticular nucleus $(\mathrm{MRN})$, periaqueductal gray (PAG), anterior pretectal nucleus (APN), nucleus of the optic tract (NOT), olivary pretectal nucleus (OP), posterior pretectal nucleus (PPT), subiculum (SUB), postsubiculum (POST, more in appendix figure 1), posteromedial visual area (VISpm) and presubiculum (PRE).

Figure 3 is an example of two anterograde projections which projected from the SC to PAG and RN/CLI. In previous studies, there are more projections from $\mathrm{SC}$ to other regions. The SC can project to lateral geniculate nucleus (LGN). Hughes et al., 1984 observed that superior colliculus projected to the LGN, and the projection cells of the SC were mainly labelled in superficial layer ( I and II, ZO and SGR), some of the labeled cells were located in the layer III (OP) [29]. Several studies showed that the projection from the superior colliculus to the lateral geniculate nucleus is relatively simple and direct [30-32]. The nerve impulse of parabigeminal nucleus ( $\mathrm{Pbg}$ ) mainly comes from the superficial layer of SC. Baleydier et al 1979 injected HRP into the Pbg of the cat, they found that there were more than $60 \%$ in the deep layers of the $\mathrm{SC}, 30 \%$ in the IGR layer, and there were about $10 \%$ of the cells in the superficial layer. In the experiments, it was confirmed that the SC had fibers connecting directly to $\mathrm{Pbg}$ [33]. It has been mentioned that the function of the $\mathrm{SC}$ is complex, and it is also involved in the physiological activities of hearing, somatosensory and other activities. The common projection site is IC [34-35].

Table 3. The projection from SC to other structures using AAV tracer based on $A B A$.

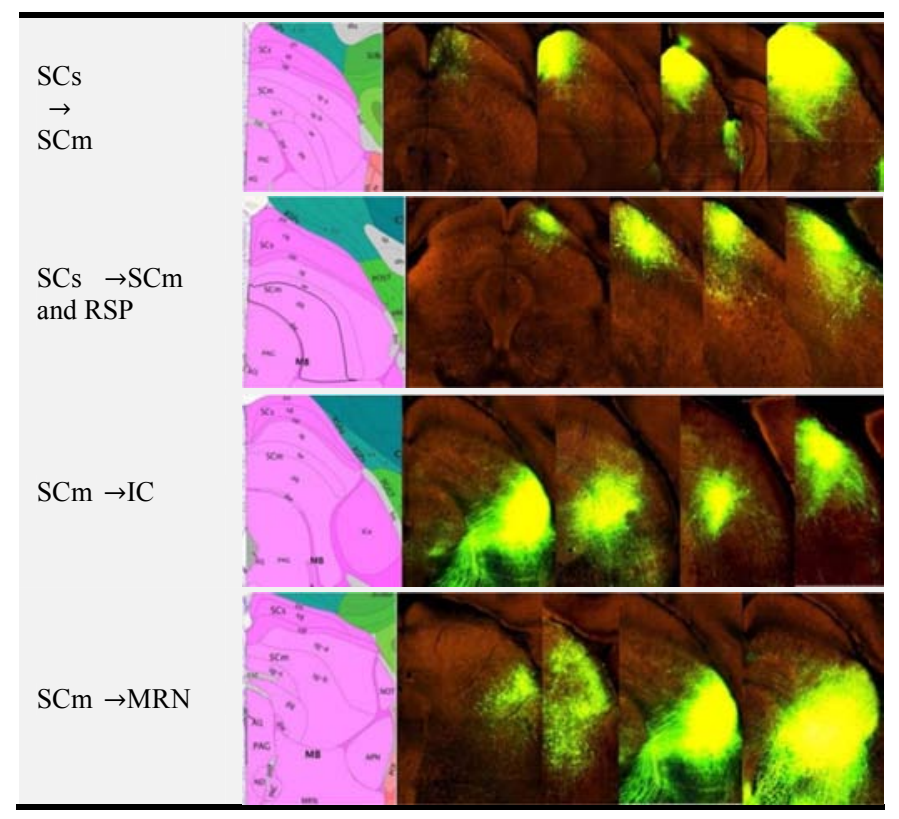



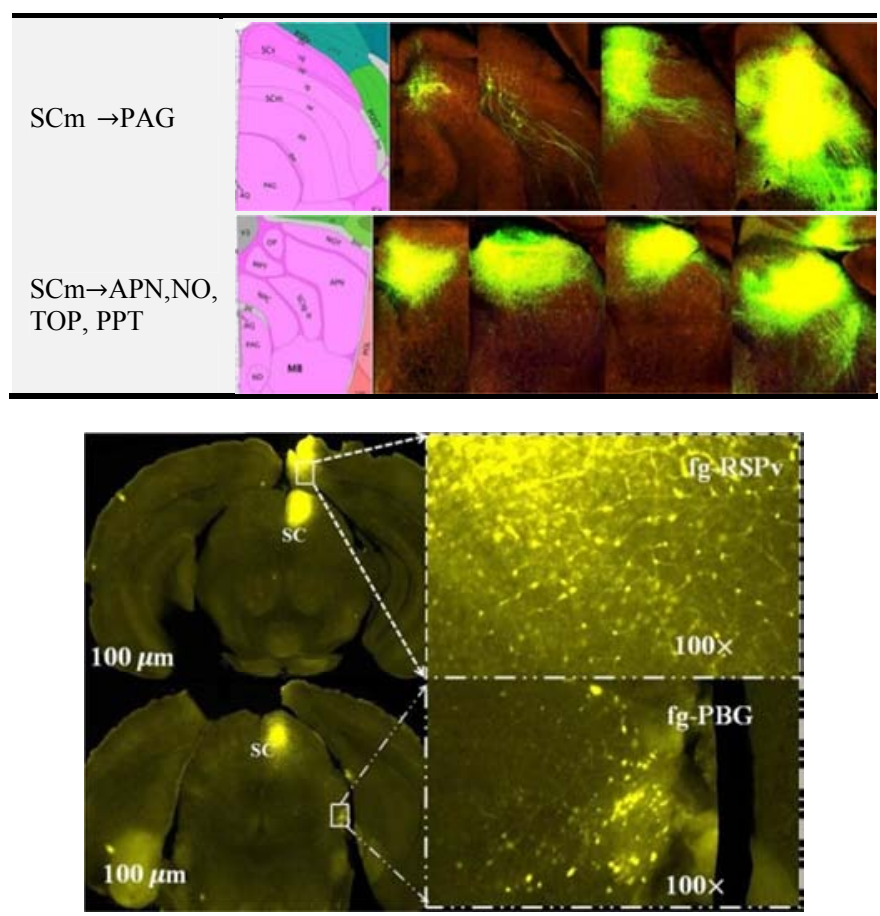

Figure 4. The retrograde projections from other regions to the SC using the fg tracer.

As shown in figure 4, the left image is composed of injection sites from other structures to the $\mathrm{SC}$, and the right image is the zoom in $(100 \%$ larger) projected structures. These two representative projections are used the fg tracer. The yellow dots illustrate that the projections from PSPv and PBG are relatively obvious. In addition, there are more small structures, which can also project to $\mathrm{SC}$ are not mentioned in the figure 4, such as Red Nucleus (RN), PAG, IC, Primary visual area (VIS), Midbrain reticular nucleus (MBN), RL, Sn, $\mathrm{Pbg}$, Rostral linear nucleus raphe (RLN), and Central linear nucleus raphe (CLI).

There are more projections from other regions that are explored in the past studies. Several studies inferred that the retina can project to $\mathrm{SC}$, since the projection from the retina to $\mathrm{SC}$ is one of the largest sources of afferent fibers in the SC [36-37]. The visual cortex (including the area of 17, the area of the 18, and the area of the 19) is mainly projected into the visual layer and the IGR layer of the SC and most of them are projected to the layer IV and V (IGR and IW) [38-39]. There is a fiber linkage between the $\mathrm{Pbg}$ and the $\mathrm{SC}$, which means $\mathrm{Pbg}$ can project to the SC and the SC can also project to the Pgb. Bickford et al., 1992 studied the projection from $\mathrm{SNr}$ to SC by HRP combining with GABA immunohistochemistry, suggested that the projection from SNR to the $\mathrm{SC}$ is GABA-ergic neurons [18]. The IC is an important relay nucleus and auditory reflex center in the auditory system and it is usually divided into three parts: the central, peripheral and central nuclei. SC and IC are collectively known as the Corpora Quadrigemina (CQ), and the pathway of the SC and IC is relatively important (appendix figure 2 showed the scheme of the anterograde and retrograde projection circuitries between SC and IC). The SC can project into the
$\mathrm{SC}$, and the IC can also project into the deep layers of the SC, which indicates that in addition to being responsible for visual stimulation, the SC are responsible for auditory and other sensory stimulation [41-43].

\section{Strengths and Weaknesses}

The strength of this paper is that it delineates for the first time the sub-regions of the SC using Nissl stain, gene expression and the different tracers based on the two online sources. However, there are still some weaknesses in this study. Firstly, this study relies on the ABA too much, and some of the information may not complete since the ABA is still not fully complete, thus it still requires more information. For example, the connectivity of SC with other structures has not been fully described in the atlas. Furthermore, the resolution of some images is still not high enough, and it is difficult to distinguish different layers. For example, it is hard to find the seven layers of SC in Nissl stain images. Future studies should concentrate on the connectivity of SC (the connectivity of SC is limited by the sources, especially in the mouseconnectome website, where only two structures can be found). There are actually more projections related to SC which are not delineated in the sources, but past studies show these projections. It may also be ideal to further explore the Tpd5211 gene in the SC, since it is densely expressed in the SC, so may play a greater role in the SC than expected.

\section{Conclusion}

The Superior Colliculus plays an important role in visual target selection and attention shifting (both overt and covert attention). In this study, it addresses the cytoarchitecure and connectivity of the SC in mouse brain. This study demonstrated that gene Tpd5211 is densely expressed in the SC, which helps delineate the borders of the SC. In addition, the anterograde and retrograde projection circuitries between SC and IC are particularly addressed, which indicated that the $\mathrm{SC}$ is involved in the visual, auditory and other somatosensory physiological activities. However, this study does not clarify the entire complexity of the SC. For future work, we will investigate more genes which is expressed in $\mathrm{SC}$, and explore more connectivities of the SC.

\section{Appendix}

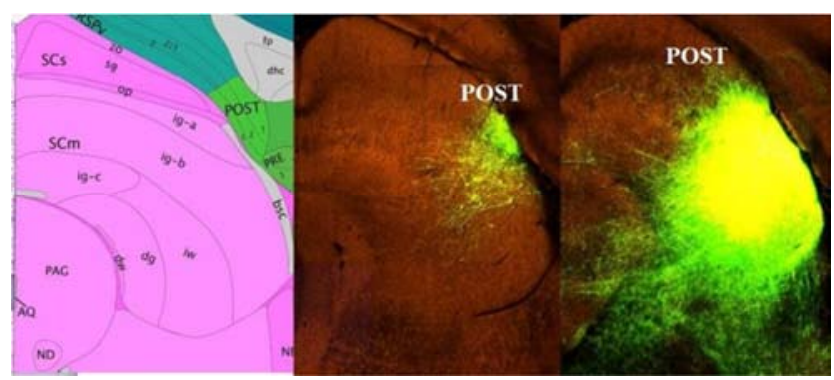

Figure 5. The projection from the SC to the POST. 


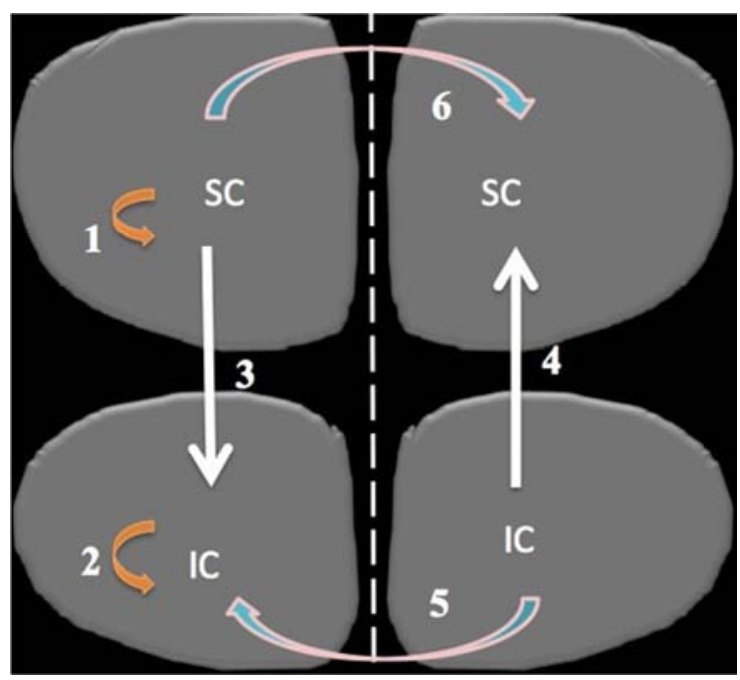

Figure 6. The projections involved in the IC and the SC (dorsal view of the surface of the corpora quadrigemina). 1: Intrinsic projections of the $S C, 2$ : Intrinsic projections of the SC, 3: Projections from the SC to the IC, 4: Projections from the IC to the SC, 5: Commissural projections of the SC, 6: Commissural projections of the IC. For easy understanding, some of the contralateral SC/ IC projections are not shown in this scheme [44].

\section{References}

[1] Inayat, S., Barchini, J., Chen, H., Feng, L., Liu, X., \& Cang, J. (2015). Neurons in the most superficial lamina of the mouse superior colliculus are highly selective for stimulus direction. Journal of Neuroscience, 35(20), 7992-8003.

[2] Huerta, M. F., Frankfurter, A., \& Harting, J. K. (1983). Studies of the principal sensory and spinal trigeminal nuclei of the rat: projections to the superior colliculus, inferior olive, and cerebellum. Journal of Comparative Neurology, 220(2), 147-167.

[3] Grantyn, R. (1987). Gaze control through superior colliculus: structure and function. Reviews of oculomotor research, 2, 273-333.

[4] Harting, J. K., Huerta, M. F., Hashikawa, T., \& van Lieshout, D. P. (1991). Projection of the mammalian superior colliculus upon the dorsal lateral geniculate nucleus: organization of tectogeniculate pathways in nineteen species. Journal of Comparative Neurology, 304(2), 275-306.

[5] Isa, T. (2002). Intrinsic processing in the mammalian superior colliculus. Current opinion in neurobiology, 12(6), 668-677.

[6] Baldwin, M. K., \& Kaas, J. H. (2012). Cortical projections to the superior colliculus in prosimian galagos (Otolemur garnetti). Journal of Comparative Neurology, 520(9), 2002-2020.

[7] Smith, P. H., Manning, K. A., \& Uhlrich, D. J. (2010). Evaluation of inputs to rat primary auditory cortex from the suprageniculate nucleus and extrastriate visual cortex. Journal of Comparative Neurology, 518(18), 3679-3700.

[8] Khibnik, L. A., Tritsch, N. X., \& Sabatini, B. L. (2014). A Direct Projection from Mouse Primary Visual Cortex to Dorsomedial Striatum. PLoS ONE, 9(8), e104501.

[9] Zhao, J., Urakawa, S., Matsumoto, J., Li, R., Ishii, Y., Sasahara, M., \& Nishijo, H. (2013). Changes in Otx2 and parvalbumin immunoreactivity in the superior colliculus in the platelet-derived growth factor receptor- $\beta$ knockout mice. BioMed research international, 2013.

[10] Hoshino, K., Horie, M., Nagy, A., Berényi, A., Benedek, G., \& Norita, M. (2010). Direct synaptic connections between superior colliculus afferents and thalamo-insular projection neurons in the feline suprageniculate nucleus: A double-labeling study with WGA-HRP and kainic acid. Neuroscience research, 66(1), 7-13.

[11] Fisher, S. D., \& Reynolds, J. N. J. (2014). The intralaminar thalamus - an expressway linking visual stimuli to circuits determining agency and action selection. Frontiers in Behavioral Neuroscience, 8, 115.

[12] Langer, T. P., \& Lund, R. D. (1974). The upper layers of the superior colliculus of the rat: a Golgi study. Journal of Comparative Neurology, 158(4), 405-435.

[13] Schneider, G. E. (1969). Two visual systems. Science.

[14] Cynader, M., \& Berman, N. (1972). Receptive-field organization of monkey superior colliculus. Journal of Neurophysiology, 35(2), 187-201.

[15] Hubel, D. H., LeVay, S., \& Wiesel, T. N. (1975). Mode of termination of retinotectal fibers in macaque monkey: an autoradiographic study. Brain research, 96(1), 25-40.

[16] Mathers, J., Benumof, J. L., \& Wahrenbrock, E. A. (1977). General anesthetics and regional hypoxic pulmonary vasoconstriction. Anesthesiology, 46(2), 111-114.

[17] Thalluri, J., \& Henry, G. H. (1989). Neurons of the striate cortex driven trans-synaptically by electrical stimulation of the superior colliculus. Vision research, 29(10), 1319-1323.

[18] Bickford, P., Heron, C., Young, D. A., Gerhardt, G. A., \& dela Garza, R. (1992). Impaired acquisition of novel locomotor tasks in aged and norepinephrine-depleted F344 rats. Neurobiology of aging, 13(4), 475-481.

[19] Roffler-Tarlov, S., Schildkraut, J. J., \& Draskoczy, P. R. (1973). Effects of acute and chronic administration of desmethylimipramine on the content of norepinephrine and other monoamines in the rat brain. Biochemical pharmacology, 22(22), 2923-2926.

[20] Harting, J. K., \& Van Lieshout, D. P. (1991). Spatial relationships of axons arising from the substantia nigra, spinal trigeminal nucleus, and pedunculopontine tegmental nucleus within the intermediate gray of the cat superior colliculus. Journal of comparative neurology, 305(4), 543-558.

[21] Redgrave, P., Mitchell, I. J., \& Dean, P. (1987). Descending projections from the superior colliculus in rat: a study using orthograde transport of wheatgerm-agglutinin conjugated horseradish peroxidase. Experimental brain research, 68(1), 147-167.

[22] Bajo, V. M., Merchán, M. A., López, D. E., \& Rouiller, E. M. (1993). Neuronal morphology and efferent projections of the dorsal nucleus of the lateral lemniscus in the rat. Journal of Comparative Neurology, 334(2), 241-262.

[23] Lein, E. S., Hawrylycz, M. J., Ao, N., Ayres, M., Bensinger, A., Bernard, A. \& Chen, L. (2007). Genome-wide atlas of gene expression in the adult mouse brain. Nature, 445(7124), 168. 
[24] Pessoa, L. (2013). The cognitive-emotional brain: From interactions to integration. MIT press.

[25] O'Leary NA, Wright MW, Brister JR, Ciufo S, Haddad D, McVeigh R, Rajput B, Robbertse B, Smith-White B, Ako-Adjei D, Astashyn A, Badretdin A, Bao Y, Blinkova O, Brover V, Chetvernin V, Choi J, Cox E, Ermolaeva O, Farrell CM, Goldfarb T, Gupta T, Haft D, Hatcher E, Hlavina W, Joardar VS, Kodali VK, Li W, Maglott D, Masterson P, McGarvey KM, Murphy MR, O'Neill K, Pujar S, Rangwala SH, Rausch D, Riddick LD, Schoch C, Shkeda A, Storz SS, Sun H, Thibaud-Nissen F, Tolstoy I, Tully RE, Vatsan AR, Wallin C, Webb D, Wu W, Landrum MJ, Kimchi A, Tatusova T, DiCuccio M, Kitts P, Murphy TD, Pruitt KD. Reference sequence (RefSeq) database at NCBI: current status, taxonomic expansion, and functional annotation. Nucleic Acids Res. 2016 Jan 4; 44 (D1): D733-45.

[26] Weber, H., Kittel-Schneider, S., Gessner, A., Domschke, K., Neuner, M., Jacob, C. P. \& Baune, B. T. (2011). Cross-disorder analysis of bipolar risk genes: further evidence of DGKH as a risk gene for bipolar disorder, but also unipolar depression and adult ADHD. Neuropsychopharmacology, 36(10), 2076-2085.

[27] https://www.ncbi.nlm.nih.gov/refseq/announcements/2015/

[28] Boue - Grabot, E., Roudbaraki, M., Bascles, L., Tramu, G., Bloch, B., \& Garret, M. (1998). Expression of GABA receptor $\rho$ subunits in rat brain. Journal of neurochemistry, 70(3), 899-907.

[29] Hughes, H. C., \& Mullikin, W. H. (1984). Brainstem afferents to the lateral geniculate nucleus of the cat. Experimental brain research, 54(2), 253-258.

[30] Fuller, G. N., \& Guiloff, R. J. (1987). Migrainous olfactory hallucinations. Journal of neurology, neurosurgery, and psychiatry, 50(12), 1688.

[31] Halverson, H. E., Hubbard, E. M., \& Freeman, J. H. (2009). Stimulation of the lateral geniculate, superior colliculus, or visual cortex is sufficient for eyeblink conditioning in rats. Learning \& Memory, 16(5), 300-307. http://doi.org/10.1101/lm.1340909

[32] Wurtz, R. H., \& Kandel, E. R. (2000). Central visual pathways. Principles of neural science, 4, 523-545.

[33] Bouwmans, G., Bigot, L., Quiquempois, Y., Lopez, F., Provino, L., \& Douay, M. (2005). Fabrication and characterization of an all-solid 2D photonic bandgap fiber with a low-loss region $(<$
$20 \mathrm{~dB} / \mathrm{km}$ ) around $1550 \mathrm{~nm}$. Optics Express, 13(21), $8452-8459$.

[34] Bartlett, E. L. (2013). The organization and physiology of the auditory thalamus and its role in processing acoustic features important for speech perception. Brain and language, 126(1), $29-48$.

[35] Bulkin, D. A., \& Groh, J. M. (2011). Systematic mapping of the monkey inferior colliculus reveals enhanced low frequency sound representation. Journal of neurophysiology, 105(4), 1785-1797.

[36] Coizet, V., Overton, P. G., \& Redgrave, P. (2007). Collateralization of the tectonigral projection with other major output pathways of superior colliculus in the rat. Journal of Comparative Neurology, 500(6), 1034-1049.

[37] Pickard, G. E., So, K. F., \& Pu, M. (2015). Dorsal raphe nucleus projecting retinal ganglion cells: Why Y cells? Neuroscience \& Biobehavioral Reviews, 57, 118-131.

[38] Shipp, S., \& Grant, S. (1991). Organization of reciprocal connections between area 17 and the lateral suprasylvian area of cat visual cortex. Visual neuroscience, 6(04), 339-355.

[39] Martinez-Conde, S., Cudeiro, J., Grieve, K. L., Rodriguez, R., Rivadulla, C., \& Acuña, C. (1999). Effects of feedback projections from area 18 layers $2 / 3$ to area 17 layers $2 / 3$ in the cat visual cortex. Journal of Neurophysiology, 82(5), $2667-2675$

[40] Stockard-Pope, J. E., Werner, S. S., \& Bickford, R. G. (1992). Atlas of neonatal electroencephalography. Raven Press.

[41] Bajo, V. M., Nodal, F. R., Bizley, J. K., \& King, A. J. (2007). The non-lemniscal auditory cortex in ferrets: convergence of corticotectal inputs in the superior colliculus. Auditory neuroanatomy: A sound foundation for sound processing, 77.

[42] Aparicio, M. A., \& Saldaña, E. (2009). Tectotectal neurons and projections: a proposal to establish a consistent nomenclature. The Anatomical Record, 292(2), 175-177.

[43] Winer, J. A., Chernock, M. L., Larue, D. T., \& Cheung, S. W. (2002). Descending projections to the inferior colliculus from the posterior thalamus and the auditory cortex in rat, cat, and monkey. Hearing research, 168(1), 181-195.

[44] Aparicio, M. A., \& Saldaña, E. (2009). Tectotectal neurons and projections: a proposal to establish a consistent nomenclature. The Anatomical Record, 292(2), 175-177. 